\title{
GUEST EDITORIAL Erectile dysfunction: risk factor or manifestation of cardiovascular disease?
}

International Journal of Impotence Research (2007) 19, 446-447; doi:10.1038/sj.ijir.3901582

Penile erection is a complex physiologic process involving cooperation between neurologic, vascular, hormonal and myogenic systems. The mechanism of erectile dysfunction (ED) is similarly multifactorial and may involve neurologic, vascular and hormonal systems, as well as psychosocial processes. There has been a great deal of recent interest in the vascular aspect of ED. Not only is treatment of vascular ED the target of phosphodiesterase-5 inhibitors (the foundation of pharmacologic treatment of ED), but also the relationship between ED and cardiovascular disease (CVD) is intriguing, as there is evidence that ED may be an omen for development of systemic cardiovascular disease and events, and may even be a manifestation of cardiovascular disease itself.

The observational evidence that describes that relationship between ED and cardiovascular disease is compelling and points to an underlying mechanism: atherosclerosis. ED and cardiovascular disease share many risk factors, including hypertension, diabetes, dyslipidemia, metabolic syndrome and age ${ }^{(1-4)}$-factors that are responsible for development of endothelial dysfunction and subsequent atherosclerosis. Solomon et al. ${ }^{5}$ investigated patients with known atherosclerosis in a study of 132 men with angiographically proven coronary artery disease. They found that the degree of ED was positively correlated with the burden of coronary atherosclerotic disease.

The clinical relevance of this relationship was investigated by Thompson, et al. ${ }^{6}$ in an analysis of a database of 8063 men without cardiovascular disease enrolled in the Prostate Cancer Prevention Trial. The prevalence or incidence of ED was associated with an increased risk of cardiovascular events, including myocardial infarction, coronary artery disease requiring intervention, stroke, transient ischemic attack, heart failure and arrhythmia (hazard ratio 1.45, confidence interval 1.25-1.69). The authors noted that the risk was comparable to several traditional cardiovascular risk factors (such as tobacco use or family history of myocardial infarction). The clinical utility of these findings is profound, as ED is a subjective symptom, which may prompt complaints and physician visits, especially as intervention has improved with the introduction of the phosphodiesterase-5 inhibitors in the late 1990s. Two studies of patients with angiographically proven coronary artery disease demonstrated that symptoms of ED often precede the diagnosis or symptoms of coronary artery disease from months to years. ${ }^{5,7}$ The presence of ED in patients, especially in those already at high cardiovascular risk, should prompt judicious evaluation for underlying systemic atherosclerotic disease. No longer is ED seen as a natural process of aging or merely a symptom. Rather, it is a potential marker for cardiovascular disease.

While the observational evidence of the relationship between ED and cardiovascular disease is compelling, it is critical to understand the underlying pathophysiologic mechanism of vascular ED to clarify whether ED is merely an epiphenomenal risk factor for CVD or is a form of CVD itself secondary to atherosclerotic disease. In the current issue of the International Journal of Impotence Research, a study reports the activity of paraoxonase (PON1) in subjects with ED. The authors found that PON1 levels were significantly lower in subjects with ED compared to age-matched controls. ${ }^{8} \mathrm{PON} 1$, a highdensity lipoprotein (HDL)-associated enzyme, has been implicated as an anti-atherogenic molecule, preventing development of systemic atherosclerosis. Its activity is correlated with HDL levels, which has been well studied and is a clinically relevant risk factor for coronary artery disease. The lower level of PON1 in patients with ED supports the premise of an underlying atherosclerotic mechanism in vascular ED. These findings may also help identify patients at increased risk of developing clinically relevant atherosclerotic disease, as the patient population in this study was strikingly young (mean age of ED group $=31$ years). While this study was limited by its cross-sectional design, further studies may help clarify the relationship among PON1, ED and atherosclerosis. Additional causative and preventative mechanisms of atherosclerosis also require investigation in patients with ED to clarify the pathophysiology of vascular ED.

Cardiovascular disease presents itself in many different forms, including coronary artery disease, cerebrovascular events and peripheral vascular disease, and they are all tied together by a common mechanism, namely atherosclerosis. If atherosclerotic disease can affect coronary, cerebral and femoral arteries without mercy, then it is logical that it can 
affect the penile artery and smaller arterioles that are key for penile tumescence. Thus, ED itself may be more than merely a risk factor for cardiovascular disease, but actually a manifestation of cardiovascular disease mediated by atherosclerosis. Still, further studies are needed to clarify the relationship between atherosclerosis and ED. Ultimately, it is important to recognize that ED is a multi-factorial disorder. Separating out the specific etiologies of ED is typically difficult, if not impossible. Thus, identification of vascular ED may not be practical at present time. In particular, up to date, it is not clear why interventions that might lead to a regression of vascular disease (if there is any at all) or drugs that might improve endothelial dysfunction do not seem to improve ED, indicating that besides a pure vascular process, other potential sources for ED, such as biochemical alterations or neuropathic changes, might play a larger than expected role in the development and maintenance of ED in general. Nevertheless, there is biochemical and observational evidence that the presence of ED should at least warrant concern for systemic atherosclerotic disease.

\section{Financial disclosure}

There is no conflict of interest.

CV Chien and ER Schwarz Division of Cardiology and Comprehensive Transplant Center, Department of Medicine, Cedars
Sinai Medical Center \& University of California Los Angeles (UCLA), Los Angeles, CA, USA

E-mail: ernst.schwarz@cshs.org

\section{References}

1 Seftel AD, Sun P, Swindle R. The prevalence of hypertension, hyperlipidemia, diabetes mellitus and depression in men with erectile dysfunction. J Urol 2004; 171: 2341-2345.

2 Johannes CB, Araujo AB, Feldman HA, Derby CA, Kleinman $\mathrm{KP}$, McKinlay JB. Incidence of erectile dysfunction in men 40 to 69 years old: longitudinal results from the Massachusetts male aging study. J Urol 2000; 163: 460-463.

3 Esposito K, Giugliano F, Martedi E, Feola G, Marfella R, D'Armiento $\mathrm{M}$ et al. High proportion of erectile dysfunction in men with the metabolic syndrome. Diabetes Care 2005; 28 : 1201-1203

4 Rodriguez JJ, Al Dashti R, Schwarz ER. Linking erectile dysfunction and coronary artery disease. Int J Impot Res 2005; 17: S12-S18.

5 Solomon H, Man JW, Wierzbicki AS, Jackson G. Relation of erectile dysfunction to angiographic coronary artery disease. Am J Cardiol 2003; 91: 23-231.

6 Thompson IM, Tangen CM, Goodman PJ, Probstfield JL, Moinpour CM, Coltman CA. Erectile dysfunction and subsequent cardiovascular disease. JAMA 2005; 294: 2996-3002.

7 Montorsi F, Briganti A, Salonia A, Rigatta P, Margonato A, Macchi A et al. Erectile dysfunction prevalence, time of onset, and association with risk factors in 300 consecutive patients with acute chest pain and angiographically documented coronary artery disease. Eur Urol 2003; 44: 360-365.

8 Ciftci H, Yeni E, Savas M, Verit A, Celik H. Paraoxonase activity in patients with erectile dysfunction. Int J Impot Res 2007; 19: 517-520 (this issue). 